\title{
Application of photodynamic therapy in gastro- intestinal disorders: an outdated or re-emerging technique?
}

\author{
Han Hee Lee ${ }^{1}$, Myung-Gyu Choi ${ }^{1}$, and Tayyaba Hasan ${ }^{2}$
}

\author{
${ }^{1}$ Division of Gastroenterology, \\ Department of Internal Medicine, \\ College of Medicine, The Catholic \\ University of Korea, Seoul, Korea; \\ ${ }^{2}$ Wellman Center for Photomedicine, \\ Massachusetts General Hospital, \\ Harvard Medical School, Boston, MA, \\ USA
}

\author{
Received: May 11, 2016 \\ Accepted: December 19, 2016

\section{Correspondence to} \\ Myung-Gyu Choi, M.D. \\ Division of Gastroenterology, \\ Department of Internal Medi- \\ cine, College of Medicine, Seoul \\ St. Mary's Hospital, The Cath- \\ olic University of Korea, 222 \\ Banpo-daero, Seocho-gu, Seoul \\ 06591, Korea \\ Tel: $+82-2-2258-2083$ \\ Fax: $+82-2-2258-2089$ \\ E-mail: choim@catholic.ac.kr
}

Photodynamic therapy (PDT) is a promising therapeutic modality that involves the administration of a photosensitizer followed by local illumination with a specific wavelength of light in the presence of oxygen. PDT is minimally invasive, has high selectivity for cancer, and has good patient compliance due to the simplicity of the procedure; therefore, PDT is widely used as a palliative and salvage treatment in patients with various gastrointestinal malignancies. When used as a salvage treatment for locoregional failures after definitive chemoradiotherapy for esophageal cancer, favorable results have been reported. PDT in conjunction with biliary stenting is a promising palliative treatment for unresectable cholangiocarcinoma, and can be used as an advanced diagnostic and therapeutic strategy in peritoneal dissemination of gastric cancer. Recent clinical reports of PDT for treating non-resectable pancreatic cancer also show promising results. To widen the application of PDT, the integration of PDT with molecular imaging and nanotechnology is being extensively studied. Based on these new developments, PDT is likely to re-emerge as a valuable technique in the treatment of diverse gastrointestinal diseases.

Keywords: Photochemotherapy; Gastrointestinal diseases; Gastrointestinal neoplasms; Palliative care; Photosensitizing agents

\section{INTRODUCTION}

Photodynamic therapy (PDT) was first introduced to treat esophageal cancer in the early 1980s [1]. When a clinical trial of PDT for the treatment of superficial esophageal cancer using porfimer sodium and a $630-\mathrm{nm}$ wavelength excimer dye laser demonstrated a noteworthy result [2], PDT was approved as a palliative therapy for superficial esophageal cancer in 1994 in Japan and 2005 in Korea, and for the palliative management of advanced esophageal cancer by the Food and Drug Administration in the United States of America in 1995.

Despite the many advantages of PDT including se- lectivity and minimal invasiveness, drawbacks resulted in a reduction of its application in gastrointestinal (GI) diseases. Like many therapeutic approaches, PDT does not allow for histologic examination of target lesions, which can be used to assess cancer invasion. In addition, without appropriate dosimetry, it can cause stricture in the GI tract. When used with Photofrin (Pinnacle Biologics, Chicago, IL, USA), the earliest and first approved photosensitizer, prolonged and potentially severe skin and ocular phototoxicity can occur. Endoscopic resection including endoscopic submucosal dissection (ESD) and endoscopic mucosal resection (EMR) have become popular and are standard treatments for superficial ear- 
ly GI neoplasia [3].

So the question arises: is PDT an outdated technique? Although the role of PDT as a curative treatment has diminished, it is still widely used as a palliative and salvage treatment because it is minimally invasive, has high selectivity for cancer, and has good patient compliance due to the simplicity of the procedure. Advanced PDT technologies that are easier to deliver to local lesions, incorporate nanoparticles, and involve new photosensitizers and light sources have been developed. One attribute of PDT is that the majority of photosensitizers currently being used are fluorescent; this enables imaging to occur during therapy. Recently, this attribute has been exploited significantly. With these new developments PDT, in combination with improvements in endoscopic equipment, may be used to treat diverse GI diseases.

In this review, we summarize previously reported uses of PDT in GI diseases and introduce more recent clinical applications.

\section{PRINCIPLES AND MECHANISMS}

PDT is a well-established clinical modality that uses light energy to destroy dysplastic and malignant lesions. It requires three core elements: light of a specific wavelength, oxygen molecules, and a photosensitizer. A photosensitizer is a molecule that can absorb light energy and transfer that energy to adjacent oxygen molecules, which initiate a photodynamic reaction by forming cytotoxic molecular species including reactive oxygen, namely singlet oxygen [4]. Because the photosensitizer is preferentially localized to target tumor cells, and areas of light illumination can be controlled, this photodynamic reaction primarily damages the neoplastic lesion and preserves the adjacent normal tissue.

The GI tract starts at the mouth and ends at the anus. Most GI cancers occur at the mucosa layer, the most exposed part of the GI wall. With the development of flexible endoscopy, light energy can be directly delivered to a malignant lesion in the GI tract. This has made PDT popular as a minimally invasive local treatment modality for GI cancer [5]. Combined with imaging for dosimetry and demarcation, the potential for its use is even greater [6].

\section{ESOPHAGEAL CANCER}

PDT has been used as an endoscopic ablative technique to eliminate Barrett's esophagus (BE) and, to a limited degree, high-grade dysplasia. This technique has resulted in regression and, in some cases, complete reversal of BE [7-10]. Since then, some studies of patients with esophageal dysplasia and cancer have reported that complete remission (CR) can be achieved in superficial lesions using PDT (Table 1) [11-19]. Studies on the use of PDT for esophageal squamous cell carcinoma (SCC) confined to the mucosal layer have suggested that PDT can be used as an alternative to esophagectomy given the low risk of lymph node metastasis in these patients. The majority of the patients included in the study had serious co-morbidities precluding surgical resection. Previous studies showed that the overall survival (OS) of patients with mucosal esophageal SCC treated with PDT was similar to that of patients who underwent esophagectomy $[20,21]$. There were no major complications and treatment-related deaths. Compared with endoscopic resection, PDT has a low complication rate, is simpler to perform, and is not limited by the size or circumference of the lesion. Given these results, PDT appears to be an ideal alternative to surgical or endoscopic resection in patients who have serious comorbidities and large or multifocal superficial SCC that cannot be completely resected endoscopically.

Recent studies have indicated a beneficial role for PDT in salvage treatment for locoregional failures after definitive chemoradiotherapy (CRT) for esophageal cancer. When locally remnant lesions are found after CRT, salvage esophagectomy should be preferentially considered as the curative treatment option. However, the approach has been associated with higher rates of postoperative mortality and morbidity compared with planned esophagectomy [22]. Although EMR may be an alternative treatment in these cases, it is limited to superficial lesions and the procedure requires highly experienced endoscopists. On the other hand, PDT can be used for superficial cancers and for palliation of more advanced esophageal SCC. In a retrospective study of 37 consecutive local failure patients treated with salvage PDT using porfimer sodium, CR occurred in 22 patients (59.5\%), the 5-year progression-free survival (PFS) rate was $20.7 \%$, and the OS rate was $36.1 \%$ during a median 


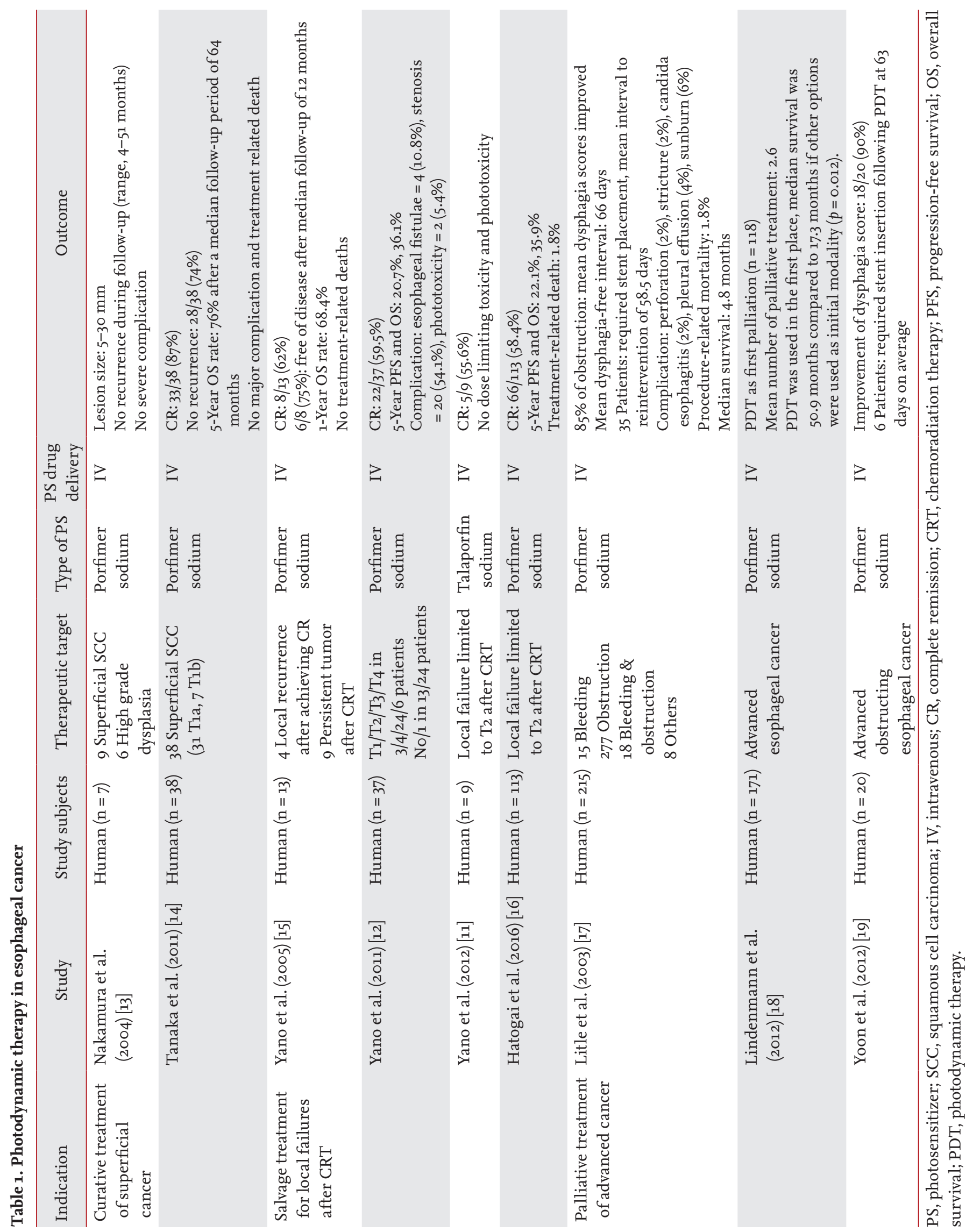


follow-up period of 55 months [12]. Another large-scale study of 113 patients who showed local failure limited to within T2 after CRT reported respective PFS and OS rates of $22.1 \%$ and $35.9 \%$, but a $1.8 \%$ rate of treatment-related death [16]. Talaporfin sodium is a second-generation, rapidly cleared photosensitizer that is expected to have less phototoxicity compared with porfimer sodium. In a phase I study, five out of nine patients with local failure after CRT achieved CR following PDT [11]. It is particularly noteworthy that phototoxicity and other severe adverse events were not observed.

PDT has also been used for palliative treatment of patients with completely or partially obstructing esophageal cancer. Two randomized controlled trials were done to compare PDT with Nd:YAG thermal ablation for palliation of dysphagia in advanced esophageal cancer $[23,24]$. Both therapies had, overall, equal efficacy in improving dysphagia, but PDT yielded a more prolonged duration of response. Another retrospective analysis was done on 250 patients who received multimodal palliative treatment, including PDT, for inoperable esophageal cancer [18]. In this study, if PDT was used as an initial therapy, the median survival was 50.9 months compared to 17.3 months if other options (endoscopic dilatation, endoluminal brachytherapy, external radiation, chemotherapy, stenting, feeding tube, or palliative resection) were used initially.

\section{CHOLANGIOCARCINOMA}

Cholangiocarcinoma (CCC) is difficult to diagnose early and usually presents at an advanced stage, which may be unresectable. Biliary stenting, mainly through the use of a self-expanding metal stent, has become the mainstay of palliative treatment for unresectable CCC with malignant strictures [25]. However, preventing tumor ingrowth and maintaining stent patency are unresolved problems [26]. Palliative options available for patients with hilar CCC include PDT, which involves a single minimally invasive treatment that has a low complication rate and is well tolerated. PDT in conjunction with biliary stenting could be an attractive treatment because it reduces the local obstruction of tumor tissue by generating singlet oxygen and may result in long-term patency.
Several uncontrolled and controlled studies comparing stenting alone to stenting with PDT for unresectable CCC have been conducted (Table 2) [27-33]. These trials showed that PDT improved cholestasis and quality of life by diminishing the need for further procedures, such as stent revision and percutaneous biliary drainage. This results in a longer stent patency period because PDT may cause tumor "remodeling," which can prolong or enhance the decompression effect. PDT was also associated with increased OS in patients with unresectable CCC. The adverse effects of PDT were predictable and relatively easy to treat. Neither study reported severe photosensitivity or procedure-related death. Thus, PDT seems to be a promising palliative treatment for unresectable CCC and should be considered part of the standard of care for these patients.

\section{PANCREATIC CANCER}

The survival statistics for pancreatic cancer (PanCa) remain dismal and the mainstay for PanCa treatment is surgery. For patients with non-resectable disease, the outlook is even more dismal. PDT can play a significant role in the management of patients with non-resectable disease. There have been two reports on the use of PDT for palliation of non-resectable PanCa [34,35]. While the two studies used different photosensitizing agents, both had promising results. The benchmark for tumor necrosis was met in both cases. In a study with verteporfin, one patient reverted to being resectable and underwent an Ro Whipple procedure [34]. Combined with other therapeutics and nanomedicine, PDT has great potential for PanCa treatment.

\section{GASTRIC CANCER}

The outcome of gastric cancer patients has dramatically improved due to advancements in the accuracy of diagnostic endoscopy, and a decrease in the invasiveness of endoscopic therapies such as EMR and ESD. However, in patients with a background of intestinal metaplasia, it is still difficult to detect malignant lesions and evaluate the resection margin. Fluorescence navigation by photodynamic diagnosis (PDD) using 5-aminolevulinic 


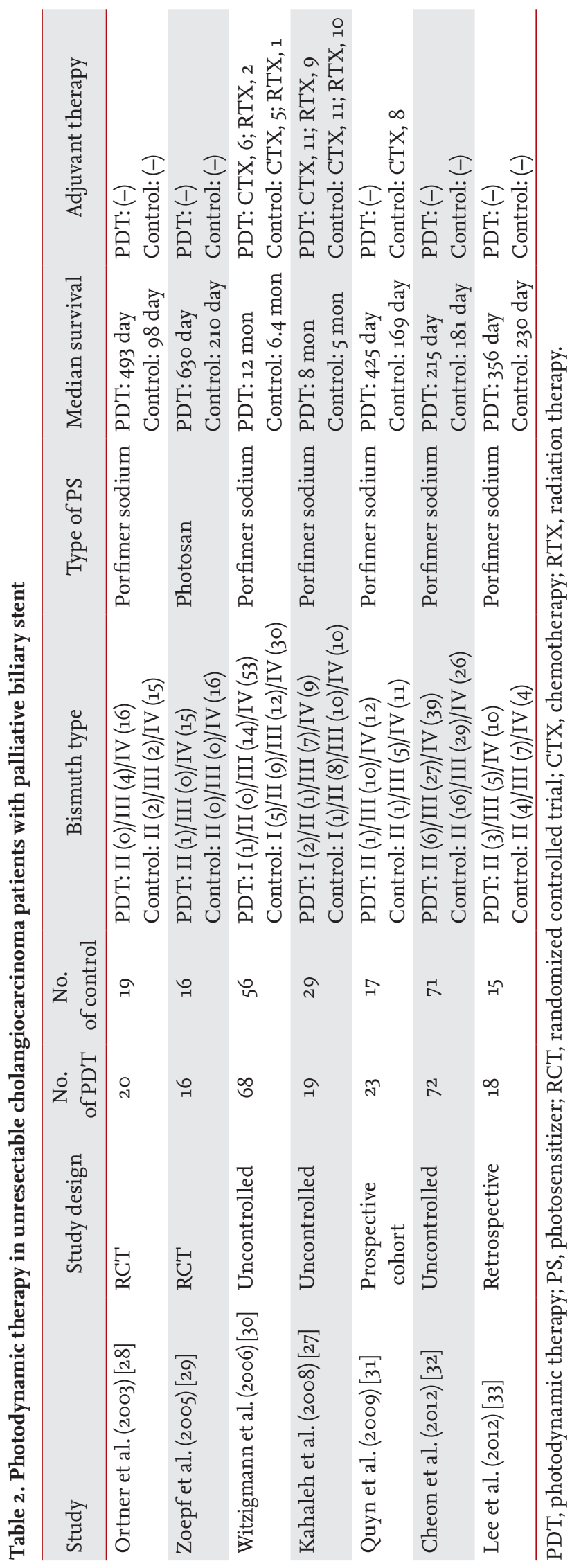

acid (ALA) can provide good visualization of gastric malignant lesions to determine the extent of the lesions. ALA is a naturally occurring amino acid and an early intermediate in the heme biosynthesis pathway. Orally administered ALA is mainly incorporated into malignant cells and metabolized to a photodynamically active protoporphyrin IX that emits a red fluorescence upon excitation with blue light [36]. The major advantage of ALA is that it is rapidly metabolized, significantly reducing the period of cutaneous photosensitivity.

Several studies have demonstrated that ALA-mediated PDD is a promising tool for diagnosing minute lesions and peritoneal metastasis in gastric cancer. A Japanese study showed that laser-based endoscopy detected upper GI tumors as red fluorescent navigation (PDD-positive) in 23 of 27 lesions [37]. Interestingly, all but one of the intestinal type carcinomas were significantly PDD-positive, whereas signet ring cell carcinomas were PDD-negative. To evaluate the detection of peritoneal metastasis in gastric cancer, ALA-PDD was added to conventional diagnostic laparoscopy for 52 advanced gastric cancer patients [38]. Using ALA-PDD, peritoneal dissemination was detected in five of 24 patients with no macroscopic evidence of peritoneal metastases on white light. In another study, staging laparoscopy with ALA-mediated PDD was performed in 13 patients with serosa-invading gastric cancer [39]. When PDD was used, the peritoneal metastasis detection rate was significantly higher than with white light. These results show that ALA-PDD may be useful for preoperative staging of advanced gastric cancer.

Treatment of advanced gastric cancer, especially in serosa-positive cases, has focused on preventing peritoneal recurrence because the peritoneum is one of the most common recurrence sites after curative gastrectomy. Although several therapeutic options, including systemic adjuvant chemotherapy and intraperitoneal chemotherapy with or without hyperthermia and CRT, have been tried [40-42], there is no standard effective treatment to prevent peritoneal recurrence and significantly increase survival. PDT may be a good alternative treatment option because micrometastatic nodules on the peritoneal surface and intraperitoneal floating cancer cells are potentially suitable PDT targets. In a study using a peritoneal metastasis mice model, talaporfin, another second-generation photosensitizer, accumulated more 
in metastatic nodules than normal organs when injected intraperitoneally [43]. Talaporfin-mediated PDT also exerts considerable antitumor effects in vivo. The total weight of peritoneal metastatic nodules was lower in mice models with PDT delivered using nanoparticles loaded with indocyanine green. These mice also had significantly improved body weight loss and a higher survival rate [44]. These results show that PDT may be a promising diagnostic and therapeutic modality in peritoneal dissemination of gastric cancer.

\section{RECENT DEVELOPMENTS TO OVERCOME THE LIMITATIONS OF PDT}

As outlined above, PDT is a very attractive treatment modality for cancer because it has minimal toxicity and can be applied repeatedly without harming adjacent normal tissue [45]. PDT is currently widely used to treat skin diseases and easily accessible malignant and pre-malignant lesions.

To maximize the effect of PDT, further research is needed to enhance its three core elements: the photosensitizer, light, and oxygen. Fig. 1 shows recent developments to overcome the limitations of PDT.

The photosensitizer can cause hypersensitivity, allergy, toxicity, and unpredictable biodistribution profiles if administrated intravenously, especially in multiple doses. Unpredictable biodistribution can lead to the accumulation of photosensitizer in easily light-exposed uninvolved tissues such as the eyes and skin, and this can result in phototoxicity and prolonged photosensitivity when patients are exposed to ambient light. Local delivery of the photosensitizer may solve these problems. Local delivery may shorten treatment time, enhance therapeutic efficacy, and eliminate skin photosensitization completely. There are several approaches for the local delivery of PDT to target GI tumors; these include spraying via the working channel of the endoscope, intratumoral injection, and the use of photosensitizer-coated stents.

To enhance the clinical use of PDT, the second challenge is to improve the targeting of the drug-activating light to the target tissue. In PDT, superficial tissues can be easily illuminated using convenient light sources, but deep tissue tumors are more difficult for light to penetrate. For example, blue light ( 400 to $450 \mathrm{~nm}$ ) penetrates approximately $1 \mathrm{~mm}$ into tissue, while orange light (590 to $620 \mathrm{~nm}$ ) penetrates approximately $1.5 \mathrm{~mm}$, and red light ( 620 to $750 \mathrm{~nm}$ ) penetrates even further, up to $3 \mathrm{~mm}$ [46]. Newer light sources, such as light-emitting diodes (LEDs), are being explored to deliver light more effectively. LEDs can generate high-energy light of desired wavelengths and can be assembled in a range of geometries and sizes [47,48]. During PDT, monitoring tumor tissue oxygenation, by measuring the partial pressure of oxygen and the level of hypoxia markers, may be used to increase the concentration of singlet oxygen to the target tissue during light irradiation. In addition, fractionating light irradiation by controlling light/dark periods can promote tissue oxygen re-perfusion to compensate for oxygen depletion caused by photochemical reactions $[49,50]$.

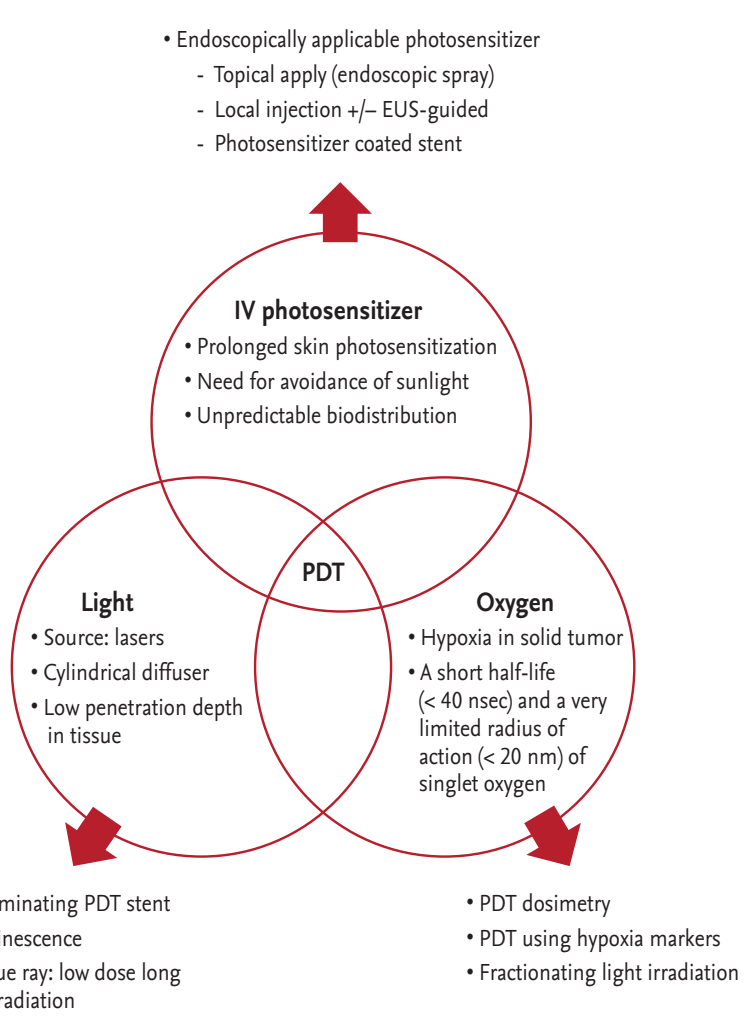

Figure 1. Recent developments and future perspectives with regard to improving the efficacy of photodynamic therapy (PDT) to treat gastrointestinal tract cancers. EUS, endoscopic ultrasound; LED, light-emitting diode. 


\section{NOVEL APPLICATIONS OF PDD AND PDT IN GI MALIGNANCY}

Much research has been done to extend the application of photodynamic techniques to the early diagnosis and treatment of GI malignancy.

In the spotlight in the field of GI endoscopy is molecular imaging because it can be used to detect lesions at an early stage. Molecular imaging can be used to visualize the molecular fingerprint of target cells, and not just their gross morphology. Developments in molecular imaging are occurring with advances in new imaging techniques such as virtual chromoendoscopy and magnifying endoscopy. In molecular imaging, disease-specific biomarkers are targeted using fluorescently labeled exogenous molecular probes including antibodies, antibody fragments, peptides, nanoparticles, and activatable probes [51]. Combining a molecular probe with a photosensitizer can enhance tumor targeting and the detection of minute lesions by the endoscopist. Furthermore, it can enable optical biopsy and theragnosis, which combines simultaneous diagnosis and therapeutics. In our group, a peptide specific to colon cancer was isolated using phage display and then conjugated to hematoporphyrin (HPP), a promising second-generation photosensitizer [52]. Immunocytochemical staining using fluorescein isothiocyanate or HPP-conjugated peptides showed high binding affinity to colon cancer cell lines. The HPP-conjugated cancer-specific peptide showed an enhanced PDT effect, compared with HPP alone. These results show that targeted PDT using peptide probes may constitute promising candidate treatments for use in the development of colon cancer diagnostics and treatments.

Photosensitizer-embedded stents allow for repeatable intraluminal PDT, which leads to regression of surrounding GI malignant tissue and extends stent patency. A recent study of a polymeric photosensitizer (pullulan acetate-conjugated pheophorbide A)-embedded self-expanding nonvascular metal stent, which permits repeatable PDT of CCC without systemic injection of the photosensitizer, reported that the stent sustained its PDT effects for at least 2 months in vitro [53]. Furthermore, in vivo testing using HCT-116 tumor-xenograft mouse models showed degradation of tumor cells and regression of xenograft tumors after laser exposure. PDT can improve the tissue penetration efficiency $\left(\mathrm{PE}_{\%}\right)$ of released drugs in non-vascular drug-eluting stents (DESs) by breaking the epithelial layer with singlet oxygen, a process called photochemical tissue penetration (PTP). We developed a PTP-DES with a photosensitizer (chlorin e6) incorporated in a gemcitabine-eluting polyurethane membrane [54]. This stent demonstrated a 2-fold increase in the $\mathrm{PE}_{\%}$ of gemcitabine, and tumor growth was effectively inhibited in an in vivo mouse model. Our results indicate that the PTP-DES system has great potential for improving the therapeutic effect of conventional DES.

Nanomedicine that combines PDT with molecular target therapy may be used to enhance the efficacy of anticancer treatments and improve outcomes for patients with highly treatment-resistant GI malignancy, while decreasing toxicity. Recently, we developed a novel photoactivatable multi-inhibitor nanoliposome (PMIL), which consists of a nanoliposome doped with a photosensitizer (benzoporphyrin derivative) in the lipid bilayer, and a nanoparticle encapsulating cabozantinib, a multikinase inhibitor [55]. This novel nanoscale drug not only causes photodynamic damage to tumor cells and microvessels but also initiates the release of cabozantinib inside the tumor. In a mouse model of PanCa, PMIL resulted in prolonged tumor reduction and metastasis suppression. These results open the door to the application of PDT in tumor-selective chemotherapy.

\section{CONCLUSIONS}

We reviewed a number of key studies that used PDT to treat GI malignancy. Drawbacks of PDT including suboptimal efficacy, phototoxicity, and high cost have curtailed the initial enthusiasm for this procedure in treating GI malignancy. PDT still has a role as a palliation and salvage treatment because it is a minimally invasive procedure. Much effort has been put into widening the application of PDT, and favorable results in areas such as early diagnosis, salvage treatment for local failures, and palliation of GI cancer, are shedding new light on the applications of PDD and PDT. Novel integration of PDT with existing technologies such as molecular imaging, DES, and nanotechnology may make PDT a feasi- 
ble and desirable option for the treatment of GI cancers.

\section{Conflict of interest}

No potential conflict of interest relevant to this article was reported.

\section{Acknowledgments}

This work was supported by the National Research Foundation of Korea (NRF) grant funded by the Korea government (Ministry of Science, ICT \& Future Planning) (NRF-2016R1C1B2015185), by a program in the Global Research and Development Center through the NRF funded by the Ministry of Science, ICT and Future Planning (NRF-2011-0031644), by a Basic Science Research Program through the NRF funded by the Ministry of Education, Science and Technology (NRF2013R1A1A2007985), by the Catholic Medical Center Research Foundation established in 2014, and by the National Institutes of Health/National Cancer Institute (NIH/NCI) of the United States of America (grant PoiCAo84203 awarded to T. Hasan).

\section{REFERENCES}

1. McCaughan JS Jr, Guy JT, Hawley P, et al. Hematoporphyrin-derivative and photoradiation therapy of malignant tumors. Lasers Surg Med 1983;3:199-209.

2. Yoshida K, Suzuki S, Mimura S, et al. Photodynamic therapy for superficial esophageal cancer: a phase III study using PHE and excimer dye laser. Gan To Kagaku Ryoho 1993;20:2063-2066.

3. Soetikno R, Kaltenbach T, Yeh R, Gotoda T. Endoscopic mucosal resection for early cancers of the upper gastrointestinal tract. J Clin Oncol 2005;23:4490-4498.

4. Dolmans DE, Fukumura D, Jain RK. Photodynamic therapy for cancer. Nat Rev Cancer 2003;3:380-387.

5. Barr H, Dix AJ, Kendall C, Stone N. Review article: the potential role for photodynamic therapy in the management of upper gastrointestinal disease. Aliment Pharmacol Ther 2001;15:311-321.

6. Pogue BW, Sheng C, Benevides J, et al. Protoporphyrin IX fluorescence photobleaching increases with the use of fractionated irradiation in the esophagus. J Biomed Opt 2008;13:034009.

7. Laukka MA, Wang KK. Initial results using low-dose pho- todynamic therapy in the treatment of Barrett's esophagus. Gastrointest Endosc 1995;42:59-63.

8. Overholt BF, Panjehpour M. Barrett's esophagus: photodynamic therapy for ablation of dysplasia, reduction of specialized mucosa, and treatment of superficial esophageal cancer. Gastrointest Endosc 1995;42:64-70.

9. Overholt BF, Panjehpour M, Haydek JM. Photodynamic therapy for Barrett's esophagus: follow-up in 100 patients. Gastrointest Endosc 1999;49:1-7.

10. Overholt BF, Lightdale CJ, Wang KK, et al. Photodynamic therapy with porfimer sodium for ablation of high-grade dysplasia in Barrett's esophagus: international, partially blinded, randomized phase III trial. Gastrointest Endosc 2005;62:488-498.

11. Yano T, Muto M, Yoshimura K, et al. Phase I study of photodynamic therapy using talaporfin sodium and diode laser for local failure after chemoradiotherapy for esophageal cancer. Radiat Oncol 2012;7:113.

12. Yano T, Muto M, Minashi K, et al. Long-term results of salvage photodynamic therapy for patients with local failure after chemoradiotherapy for esophageal squamous cell carcinoma. Endoscopy 2011;43:657-663.

13. Nakamura T, Fukui H, Shirakawa K, Fujii Y, Fujimori T, Terano A. Photodynamic therapy of superficial esophageal cancer with a transparent hood. Gastrointest Endosc 2004;60:120-124.

14. Tanaka T, Matono S, Nagano T, et al. Photodynamic therapy for large superficial squamous cell carcinoma of the esophagus. Gastrointest Endosc 2011;73:1-6.

15. Yano T, Muto M, Minashi K, Ohtsu A, Yoshida S. Photodynamic therapy as salvage treatment for local failures after definitive chemoradiotherapy for esophageal cancer. Gastrointest Endosc 2005;62:31-36.

16. Hatogai K, Yano T, Kojima T, et al. Salvage photodynamic therapy for local failure after chemoradiotherapy for esophageal squamous cell carcinoma. Gastrointest Endosc 2016;83:1130-1139.

17. Litle VR, Luketich JD, Christie NA, et al. Photodynamic therapy as palliation for esophageal cancer: experience in 215 patients. Ann Thorac Surg 2003;76:1687-1692.

18. Lindenmann J, Matzi V, Neuboeck N, et al. Individualized, multimodal palliative treatment of inoperable esophageal cancer: clinical impact of photodynamic therapy resulting in prolonged survival. Lasers Surg Med 2012;44:189198.

19. Yoon HY, Cheon YK, Choi HJ, Shim CS. Role of photody- 
namic therapy in the palliation of obstructing esophageal cancer. Korean J Intern Med 2012;27:278-284.

20. Pennathur A, Farkas A, Krasinskas AM, et al. Esophagectomy for Ti esophageal cancer: outcomes in 100 patients and implications for endoscopic therapy. Ann Thorac Surg 2009;87:1048-1054.

21. Tachibana M, Hirahara N, Kinugasa S, Yoshimura H. Clinicopathologic features of superficial esophageal cancer: results of consecutive 100 patients. Ann Surg Oncol 2008;15:104-116.

22. Miyata H, Yamasaki M, Takiguchi S, et al. Salvage esophagectomy after definitive chemoradiotherapy for thoracic esophageal cancer. J Surg Oncol 2009;100:442446.

23. Heier SK, Rothman KA, Heier LM, Rosenthal WS. Photodynamic therapy for obstructing esophageal cancer: light dosimetry and randomized comparison with Nd:YAG laser therapy. Gastroenterology 1995;109:63-72.

24. Lightdale CJ, Heier SK, Marcon NE, et al. Photodynamic therapy with porfimer sodium versus thermal ablation therapy with Nd:YAG laser for palliation of esophageal cancer: a multicenter randomized trial. Gastrointest Endosc 1995;42:507-512.

25. Soderlund C, Linder S. Covered metal versus plastic stents for malignant common bile duct stenosis: a prospective, randomized, controlled trial. Gastrointest Endosc 2006;63:986-995.

26. O'Brien S, Hatfield AR, Craig PI, Williams SP. A three year follow up of self expanding metal stents in the endoscopic palliation of longterm survivors with malignant biliary obstruction. Gut 1995;36:618-621.

27. Kahaleh M, Mishra R, Shami VM, et al. Unresectable cholangiocarcinoma: comparison of survival in biliary stenting alone versus stenting with photodynamic therapy. Clin Gastroenterol Hepatol 2008;6:290-297.

28. Ortner ME, Caca K, Berr F, et al. Successful photodynamic therapy for nonresectable cholangiocarcinoma: a randomized prospective study. Gastroenterology 2003;125:1355-1363.

29. Zoepf T, Jakobs R, Arnold JC, Apel D, Riemann JF. Palliation of nonresectable bile duct cancer: improved survival after photodynamic therapy. Am J Gastroenterol 2005;100:2426-2430.

30. Witzigmann H, Berr F, Ringel U, et al. Surgical and palliative management and outcome in 184 patients with hilar cholangiocarcinoma: palliative photodynamic therapy plus stenting is comparable to $\mathrm{r} 1 / \mathrm{r} 2$ resection. Ann Surg 2006;244:230-239.

31. Quyn AJ, Ziyaie D, Polignano FM, Tait IS. Photodynamic therapy is associated with an improvement in survival in patients with irresectable hilar cholangiocarcinoma. HPB (Oxford) 2009;11:570-577.

32. Cheon YK, Lee TY, Lee SM, Yoon JY, Shim CS. Longterm outcome of photodynamic therapy compared with biliary stenting alone in patients with advanced hilar cholangiocarcinoma. HPB (Oxford) 2012;14:185-193.

33. Lee TY, Cheon YK, Shim CS, Cho YD. Photodynamic therapy prolongs metal stent patency in patients with unresectable hilar cholangiocarcinoma. World J Gastroenterol 2012;18:5589-5594.

34. Huggett MT, Jermyn M, Gillams A, et al. Phase I/II study of verteporfin photodynamic therapy in locally advanced pancreatic cancer. Br J Cancer 2014;110:1698-1704.

35. Bown SG, Rogowska AZ, Whitelaw DE, et al. Photodynamic therapy for cancer of the pancreas. Gut 2002;50:549-557.

36. Kelty CJ, Brown NJ, Reed MW, Ackroyd R. The use of 5-aminolaevulinic acid as a photosensitiser in photodynamic therapy and photodiagnosis. Photochem Photobiol Sci 2002;1:158-168.

37. Isomoto $\mathrm{H}$, Nanashima $\mathrm{A}$, Senoo $\mathrm{T}$, et al. In vivo fluorescence navigation of gastric and upper gastrointestinal tumors by 5 -aminolevulinic acid mediated photodynamic diagnosis with a laser-equipped video image endoscope. Photodiagnosis Photodyn Ther 2015;12:201-208.

38. Kishi K, Fujiwara Y, Yano M, et al. Diagnostic laparoscopy with 5-aminolevulinic-acid-mediated photodynamic diagnosis enhances the detection of peritoneal micrometastases in advanced gastric cancer. Oncology 2014;87:257265 .

39. Kishi K, Fujiwara Y, Yano M, et al. Staging laparoscopy using ALA-mediated photodynamic diagnosis improves the detection of peritoneal metastases in advanced gastric cancer. J Surg Oncol 2012;106:294-298.

40. Mari E, Floriani I, Tinazzi A, et al. Efficacy of adjuvant chemotherapy after curative resection for gastric cancer: a meta-analysis of published randomised trials. A study of the GISCAD (Gruppo Italiano per lo Studio dei Carcinomi dell'Apparato Digerente). Ann Oncol 2000;11:837843.

41. Yan TD, Black D, Sugarbaker PH, et al. A systematic review and meta-analysis of the randomized controlled trials on adjuvant intraperitoneal chemotherapy for re- 
sectable gastric cancer. Ann Surg Oncol 2007;14:2702-2713.

42. Baeza MR, Giannini TO, Rivera SR, et al. Adjuvant radiochemotherapy in the treatment of completely resected, locally advanced gastric cancer. Int J Radiat Oncol Biol Phys 2001;50:645-650.

43. Kishi K, Yano M, Inoue M, et al. Talaporfin-mediated photodynamic therapy for peritoneal metastasis of gastric cancer in an in vivo mouse model: drug distribution and efficacy studies. Int J Oncol 2010;36:313-320.

44. Tsujimoto H, Morimoto Y, Takahata R, et al. Photodynamic therapy using nanoparticle loaded with indocyanine green for experimental peritoneal dissemination of gastric cancer. Cancer Sci 2014;105:1626-1630.

45. Miller JD, Baron ED, Scull H, et al. Photodynamic therapy with the phthalocyanine photosensitizer Pc 4: the case experience with preclinical mechanistic and early clinical-translational studies. Toxicol Appl Pharmacol 2007;224:290-299.

46. Barolet D. Light-emitting diodes (LEDs) in dermatology. Semin Cutan Med Surg 2008;27:227-238.

47. Juzeniene A, Juzenas P, Ma LW, Iani V, Moan J. Effectiveness of different light sources for 5-aminolevulinic acid photodynamic therapy. Lasers Med Sci 2004;19:139-149.

48. Mang TS. Lasers and light sources for PDT: past, present and future. Photodiagnosis Photodyn Ther 2004;1:43-48.

49. Henderson BW, Busch TM, Vaughan LA, et al. Photofrin photodynamic therapy can significantly deplete or preserve oxygenation in human basal cell carcinomas during treatment, depending on fluence rate. Cancer Res 2000;60:525-529.

50. Curnow A, Haller JC, Bown SG. Oxygen monitoring during 5-aminolaevulinic acid induced photodynamic therapy in normal rat colon. Comparison of continuous and fractionated light regimes. J Photochem Photobiol B 2000;58:149-155.

51. Goetz M, Wang TD. Molecular imaging in gastrointestinal endoscopy. Gastroenterology 2010;138:828.e1-833.e1.

52. Kim JH, Roh YJ, Kim IW, et al. Targeted photodynamic therapy with colon cancer-specific peptide conjugated photosensitizer. Photodiagnosis Photodyn Ther 2015;12:340.

53. Bae BC, Yang SG, Jeong S, et al. Polymeric photosensitizer-embedded self-expanding metal stent for repeatable endoscopic photodynamic therapy of cholangiocarcinoma. Biomaterials 2014;35:8487-8495.

54. Min D, Jeong D, Choi MG, Na K. Photochemical tissue penetration via photosensitizer for effective drug penetration in a non-vascular tumor. Biomaterials 2015;52:484493.

55. Spring BQ, Bryan Sears R, Zheng LZ, et al. A photoactivable multi-inhibitor nanoliposome for tumour control and simultaneous inhibition of treatment escape pathways. Nat Nanotechnol 2016;11:378-387. 